\title{
eJRIEPS
}

Ejournal de la recherche sur l'intervention en éducation physique et sport

$35 \mid 2015$

Varia

\section{Les dimensions personnelles de gestion du risque d'étudiants en écoles supérieures de cirque : une étude exploratoire}

\section{Delphine Lafollie}

\section{(2) OpenEdition}

\section{Édition électronique}

URL : http://journals.openedition.org/ejrieps/1517

DOI : $10.4000 /$ ejrieps. 1517

ISSN : 2105-0821

\section{Éditeur}

ELLIADD

Référence électronique

Delphine Lafollie, «Les dimensions personnelles de gestion du risque d'étudiants en écoles supérieures de cirque : une étude exploratoire », eJRIEPS [En ligne], 35 | 2015, mis en ligne le 01 avril 2015, consulté le 03 octobre 2019. URL : http://journals.openedition.org/ejrieps/1517 ; DOI : 10.4000/ ejrieps. 1517

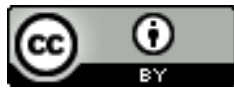

La revue eJRIEPS est mise à disposition selon les termes de la Creative Commons Attribution 4.0 International License. 


\section{eJRIEPS 35 avril 2015}

\section{Les dimensions personnelles de gestion du risque d'étudiants en écoles} supérieures de cirque : une étude exploratoire

\section{Delphine Lafollie}

Université de Reims Champagne-Ardenne, CEREP EA 4692, Reims, France

\section{Résumé}

La gestion du risque dans le cirque a surtout été étudiée d'un point de vue sociologique qui met largement en avant la "gestion des émotions " (Fourmaux, 2006) ou l'importance des «dimensions personnelles, psychologiques» (Legendre, 2014). Notre étude exploratoire, constituée de 30 entretiens semi-directifs d'étudiants en écoles supérieures de cirque, vise une compréhension plus approfondie de ces dimensions personnelles à la lumière de données issues de la psychologie du sport appliquée. Les résultats mettent en avant des dimensions intra-individuelles de gestion du risque (gestion de la peur, confiance en soi et dans son corps, et concentration) et des dimensions interindividuelles (forte confiance accordée à l'enseignant ou aux partenaires, émulation du groupe). L'apprentissage ou le développement de la plupart de ces dimensions personnelles passe indirectement par la maitrise technique issue d'apprentissages formels répétitifs et progressifs. Les techniques mentales plus spécifiques concernant la gestion de la peur ou la concentration sont, par contre, plutôt issues d'un apprentissage personnel et informel, qui semble pertinent, mais le serait probablement encore plus s'il était formalisé.

Mots clés : risque, cirque, psychologie du sport, techniques mentales, maîtrise technique

\section{Introduction}

La question du risque et des prises de risque dans la société et dans le sport notamment est particulièrement étudiée. A ce titre, des déterminants biologiques (e.g., Zuckerman, 2006), psychologiques (e.g., Michel, 2001) ou sociologiques (e.g., Soulé \& Corneloup, 2007) sont notamment mis en avant dans des études émanant de différents champs disciplinaires. Cependant, la gestion du risque, en particulier en contexte sportif, a fait l'objet de beaucoup moins de recherche. Dans le domaine du cirque professionnel, cette question est pourtant centrale : la carrière professionnelle des circassiens est directement dépendante de la préservation de leur intégrité corporelle et donc de la gestion qu'ils 


\section{eJRIEPS 35 avril 2015}

feront de leurs prises de risque. Or, paradoxalement, dans le monde du cirque contemporain ce risque est euphémisé (Fourmaux, 2006 ; Goudart, 2002 ; Sizorn, 2008). Cette particularité se retrouve de façon encore plus surprenante dans les écoles supérieures de cirque en France qui forment à bac +3 les artistes de cirque de demain : "Parler du risque et de gestion dans les écoles professionnelles de cirque ne semble donc pas si évident, alors que cette dimension apparait clairement dans les maquettes pédagogiques et dans les discours des professionnels des écoles. Souvent le risque est banalisé, encore une fois euphémisé dans une vision plutôt fataliste » (Legendre, 2014, p.5). Dans ce contexte, nous pouvons alors nous demander quelle gestion du risque et surtout quels apprentissages de cette gestion du risque vont être mis en œuvre dans ces écoles ? Cette étude exploratoire qualitative va tenter d'apporter des éléments de réponse à ces questions, à travers une recherche menée auprès d'étudiants d'écoles supérieures de cirque en France. Nous mettrons donc en avant quelles gestions du risque émanent du discours de ces étudiants et quels apprentissages ou quels types d'apprentissage (formel, informel) en découlent.

Dans les activités circassiennes professionnelles, le risque est pluriel. Dans les discours des enseignants et des enseignés des écoles professionnelles de cirque, plusieurs types de risque émergent (Legendre, 2014): tout d'abord le risque corporel avec des conséquences à court terme ou à long terme, et dans une moindre mesure, le risque dans une activité de représentation («perdre la face », ne pas réussir lors d'un spectacle), le risque de l'insertion professionnelle puis des conditions économiques d'exercice du métier, et enfin le risque dans les activités de création («lâcher prise » et donc oser en terme de création). Dans le cadre de cette étude, nous nous limiterons à la gestion du risque corporel. Par ailleurs, la gestion du risque est plurielle également, nous avons choisi de nous focaliser sur l'importance des dimensions personnelles dans cette gestion. Par conséquent, notre recherche sera dans un premier temps contextualisée avec quelques éléments théoriques concernant tout d'abord le risque, sa gestion et son apprentissage en contexte sportif et en écoles de cirque. Puis nous présenterons le cadre utilisé pour définir les dimensions personnelles. Nous expliciterons ensuite la méthodologie de cette étude. Enfin, les résultats seront présentés et discutés à la lumière des données scientifiques relatives aux déterminants de la performance sportive et à l'intervention psychologique dans le sport, cette dernière référence permettra notamment de mettre en avant quelques pistes pour la formation. 


\section{eJRIEPS 35 avril 2015}

\section{Gestion du risque en contexte sportif et en école supérieure de cirque}

2.1. Risque et prise de risque en contexte sportif

Le risque, tout d'abord, renvoie à l'éventualité d'un événement incertain qui peut conduire à un dommage, à une perte. Deux éléments sont donc indissociables du risque : l'imprévisibilité et les conséquences négatives de l'événement. Collard (2002) évoque ainsi le hasard et les enjeux (ce qui est misé en début ou en cours de partie, que le joueur tente de ne pas perdre) qui peuvent être compétitifs mais surtout corporels dans les sports à risque (quand l'exécution d'une action motrice, prévue explicitement par le code du jeu, peut affecter l'intégrité physique).

La prise de risque peut être définie comme « la participation active de l'individu dans un comportement pouvant être dangereux » (Michel, Purper-Ouakil \& Mouren-Siméoni, 2002, p.584). C'est une « décision impliquant un choix qui se caractérise par un certain degré d'incertitude quant aux probabilités d'échec ou de réussite. A chaque probabilité est associée une utilité, un bénéfice du risque » (ibid., p.584). La prise de risque comporte ainsi quatre caractéristiques : deux relevant du risque (l'incertitude du résultat, et les conséquences de l'action éventuellement négatives) et deux relevant du choix personnel de l'individu qui va prendre des risques (le choix intentionnel et volontaire de s'engager, et l'objectif positif, la récompense recherchée dans cet engagement). Les bénéfices escomptés dans les prises de risque sportives sont généralement d'ordre personnel : estime de soi, réputation personnelle, virilité, courage (Raveneau, 2006), reconnaissance sociale, identité, bien-être, mérite, plaisir (Corneloup \& Soulé, 2002), recherche de sensations, de limites ou d'autonomie, construction de soi, accomplissement de soi (Soulé \& Corneloup, 1998). II a été notamment mis en avant en psychologie du sport, deux grands profils de preneurs de risque sportif (Lafollie \& Le Scanff, 2008): les compensateurs surtout à la recherche d'une valorisation ou d'une construction de soi et les hédonistes à la recherche de sensations fortes, de plaisir. « Le risque devient alors l'élément d'un mode de vie placé sous le signe du jeu, du ressenti. La pratique d'un sport à risque, au sens premier du terme, rentre a priori dans ce cadre (...), prise de risque comme possibilité d'atteindre une certaine harmonie » (Soulé \& Corneloup, 1998, p.8-9). En contexte sportif, la prise de risque est donc avant tout considérée comme constructrice avant d'être éventuellement destructrice (accident).

Ce choix volontaire de s'engager dans l'activité va être largement modulé par la représentation du risque encouru qui diffère selon les individus. En effet, le risque est fortement subjectif. II correspond avant tout à une évaluation personnelle de la situation 


\section{eJRIEPS 35 avril 2015}

(Delignières, 1993), même si certains critères qui relèvent du risque objectif (variable quantitative qui caractérise le contexte de la tâche prenant en compte la probabilité d'accident et sa gravité, Delignières, 1991) peuvent être dégagés en lien notamment avec la logique interne des activités physiques et sportives (Collard, 1998). Le risque sera donc pris si l'individu y voit plus de bénéfices, d'intérêts que de coûts négatifs. Dans la théorie homéostatique du risque (Wilde, 1988, 2012), la prise de risque dépend de la comparaison entre le risque préférentiel ou risque cible (meilleur compromis entre les coûts et les bénéfices des actions possibles) qui est un standard spécifique à chacun (niveau optimal de risque que l'on souhaite prendre selon le contexte) et le risque perçu (évaluation de la dangerosité actuelle) (Delignières, 1993). L'individu tendra alors à réduire, de façon intuitive, les écarts entre le risque préférentiel et le risque perçu en modulant le risque pris : risque élevé si le risque perçu est inférieur au risque préférentiel, risque faible si le risque perçu est supérieur au risque préférentiel (Wilde, 1988).

La prise de risque va donc dépendre des évaluations subjectives, et également intuitives, du risque préférentiel et du risque perçu qui sont liées à de nombreux facteurs. En contexte sportif, de multiples biais, imprévus et données culturelles vont, en effet, modifier le rapport préférentiel au risque : pression des médias, logique de la distinction, valeurs affectionnées, dynamique et ambiance de groupe, état psychologique, situation anomique (Corneloup \& Soulé, 2002). Par ailleurs, des recherches en psychologie du sport montrent aussi, dans une perspective différentielle, que des caractéristiques individuelles impactent le risque subjectif et l'engagement dans une situation risquée. Ces caractéristiques sont en particulier émotionnelles (e.g., alexithymie: incapacité à identifier, différencier, verbaliser et communiquer ses émotions ou sentiments) ou liées à la personnalité, comme la recherche de sensations, l'extraversion ou l'impulsivité (e.g., Bonnet, Pedinielli, Romain \& Rouan, 2003 ; Lafollie \& Le Scanff, 2007, 2008 ; Michel, 2001). Enfin, l'évaluation du risque perçu pourra dépendre des compétences perceptives de l'individu (Wilde, 2012), de variables cognitives comme la capacité de traitement de l'information, les connaissances ou le niveau d'information sur la situation (Kouabenan, 2006) ou de façon plus générale de l'expertise des individus (Delignières, 1993). Finalement, pour Kouabenan (2006) l'évaluation subjective du risque est liée à certaines caractéristiques du risque et aux variables individuelles, psychosociologiques ou cognitives de l'individu.

Agir sur le risque subjectif (préférentiel et perçu), qui détermine la prise de risque, semble ainsi difficile : il serait intuitif, donc peu rationalisable, et sous tendu par un grand nombre de variables en interaction. Plutôt que de jouer sur l'engagement dans une situation 


\section{eJRIEPS 35 avril 2015}

risquée qui parait alors difficilement influençable, la gestion du risque pourrait être basée sur une augmentation de la sécurité passive (amélioration des matériaux, des dispositifs de sécurité): en cas d'accident, les conséquences corporelles seraient amoindries. Cependant, si cette solution s'avère efficace à court terme, elle ne l'est pas à long terme. En effet, la sécurité passive n'influence pas le niveau de risque préférentiel, mais diminue le risque perçu (évaluation de la dangerosité de la situation). De ce fait, dans un but de « conservation du risque » (Wilde, 1988), les individus percevant la situation comme moins risquée, vont prendre plus de risque pour être en phase avec leur standard de risque préférentiel. Cette théorie est connue dans le domaine de la sécurité routière ou de l'alpinisme où l'introduction de dispositifs plus sécurisants (respectivement $A B S$ ou descendeurs métalliques, piolets-traction, etc.) a augmenté les prises de risque et les accidents (Delignières, 1993). «Plus on évolue dans un environnement sûr, plus on sera prêt à prendre des risques, et inversement »(Camiolo, 2013, p.160).

Par conséquent, la gestion des risques ne peut pas s'appuyer uniquement sur des mesures d'amélioration de la sécurité passive. En amont de l'action, elle ne pourra jouer qu'à minima sur le risque préférentiel, difficilement atteignable car infraconscient, mais elle pourra porter plus facilement sur une amélioration de l'évaluation précise de la dangerosité de la situation (risque perçu) ou sur une mise en place de stratégies logistiques (Corneloup \& Soulé, 2002). Une fois l'action engagée, la gestion des risques va concerner la mise en jeu de diverses compétences de l'individu pour assurer un déroulement de l'action en toute sécurité : mise en place, par exemple, de stratégies d'action (ibid.) et en cas de problème de stratégies sécuritaires (ibid.) ou d'habiletés d'évitement (Delignières, 1991, 1993). Nous allons détailler ces éléments de la gestion du risque et son apprentissage en contexte sportif.

\subsection{Gestion des risques et son apprentissage en contexte sportif}

Si beaucoup de recherches traitent des déterminants et des conséquences de la prise de risque dans le sport, très peu s'intéressent directement à la gestion de ces risques et encore moins à son apprentissage.

Corneloup et Soulé (2002) font partie des rares chercheurs qui évoquent la gestion des risques en contexte sportif (et plus précisément en environnement naturel). Ces sociologues s'intéressent à la manière dont se prennent les décisions dans les sports de plein air selon une approche globale. Cette dernière prend en compte les facteurs techniques, humains et organisationnels dans l'étude des risques et non la seule décision rationnelle de l'acteur (comme le fait l'approche cognitive dans la théorie des jeux 


\section{eJRIEPS 35 avril 2015}

notamment). En effet, l'environnement physique et social serait tout aussi menaçant que le comportement du pratiquant dans le déclenchement du processus du danger. Dans les sports de plein air, où existe une forte incertitude, la décision loin d'être rationnelle, va reposer sur l'expérience, des compétences assimilées, des savoirs implicites, des probabilités subjectives, des suppositions, des habitudes ou encore l'observation du comportement d'autrui. Le pratiquant utilise donc un «bricolage interactionnel » (ibid.) pour apprécier le niveau de danger et prendre des décisions satisfaisantes. La gestion des risques des sports de plein air va alors se présenter comme une interaction entre maitrise de la logistique, de la culture d'action et de la sécurité pour faire face aux multiples incertitudes rencontrées : le pratiquant va mettre en place des stratégies qui vont orienter sa décision face aux nombreux choix se présentant à lui. Tout d'abord en amont, face aux incertitudes logistiques qui peuvent être individuelles, organisationnelles, relationnelles ou technologiques, des stratégies logistiques peuvent être adoptées : que dois-je emmener ? Quelle est la fiabilité de la météo ? Quel choix du site, de la structure professionnelle ? etc. (ibid.). Ensuite dans l'action, les incertitudes peuvent être individuelles (faute technique, mauvaise stratégie d'action, etc.), environnementales (orage, avalanche, etc.), technologiques (rupture de corde, etc.), relationnelles (faute d'un partenaire, conflits, etc.) ou structurelles (faute d'un guide, matériel de location défectueux, etc.). Différentes stratégies d'action émergent alors : ai-je le niveau pour faire cette voie? Mon partenaire est fatigué, que faisons-nous ? Puis-je faire confiance à ce guide ?, etc. (ibid.). Enfin en cas de problème et face à des incertitudes individuelles (méconnaissance des techniques de réchappe, de l'utilisation d'un arva, etc.), relationnelles, structurelles ou technologiques (impossibilité de joindre les secours), des stratégies sécuritaires seront nécessaires : en cas de problème, saurai-je faire face à la situation? Ai-je bien vérifié la compétence sécuritaire de mon partenaire ? etc (ibid.).

La réflexivité du pratiquant et les méthodes pratiques utilisées sont donc incontournables dans la façon dont il va organiser son action et prendre des décisions pour assurer sa sécurité et celle des autres. Le pratiquant met alors en place différentes stratégies face aux multiples choix qui s'offrent à lui, en amont, pendant l'action et éventuellement en aval. De ce fait, pour Corneloup et Soulé (2002) une «pédagogie du risque » efficace doit reposer autant sur la maitrise logistique et la maitrise d'action que sur la maitrise sécuritaire. Cependant, la réflexion de ces sociologues ne concerne pas directement l'apprentissage de la gestion du risque. Delignières (1991, 1993), est l'un des rares auteurs qui a proposé quelques pistes sur ce sujet. En s'appuyant principalement sur un 


\section{eJRIEPS 35 avril 2015}

autre cadre (théories cognitivistes présentées précédemment), il émet des hypothèses concernant une "didactique de la sécurité » en contexte scolaire en insistant aussi sur le développement concomitant d'habiletés préventives (logistique),d' habiletés sportives et d'habiletés d'évitement pour réagir en cas de problème.

Tout d'abord, cet auteur explique que de façon générale si le risque préférentiel est difficilement affecté par des injonctions raisonnées, les psychosociologues indiquent que pour changer l'attitude des sujets envers les situations risquées, il faut déjà modifier les comportements (par la contrainte ou la répression éventuellement). Ensuite, la justification de ces nouveaux comportements moins risqués modifierait l'attitude, qui deviendrait le soubassement des comportements ultérieurs. Le risque préférentiel et donc la prise de risque pourraient alors être diminués de cette façon, surtout utilisée en sécurité routière. Concernant le risque perçu, l'évaluation précise de la dangerosité de la situation est également indispensable à la gestion des risques : une sous-évaluation des risques réels entrainera des comportements dangereux. Or il a été montré que l'expertise permet une évaluation précise et plus objective des risques. En termes d'apprentissage, il serait donc nécessaire de confronter les sujets à des situations à risque réel et leur permettre l'acquisition de compétences significatives dans leur maitrise (Delignières, 1993).

Acquérir une compétence solide dans une activité donnée est donc une des bases de la gestion des risques. Dans le contexte particulier de l'éducation physique et sportive (EPS), il s'agit de s'appuyer sur une activité sportive culturellement porteuse d'une problématique de sécurité et qui nécessite la mise en jeu d'habiletés de sécurité. Ces dernières doivent être développées, de concert avec les habiletés sportives, en situation signifiante risquée et non «à vide ». De ce fait, il faut favoriser l'apprentissage en situation des habiletés d'évitement, c'est-à-dire des tactiques d'urgence (réchappes) du sujet lui-même ou du rôle des partenaires (parade en gymnastique, assurage en escalade) pour réagir efficacement en cas de problème. II faut aussi permettre le développement des compétences nécessaires pour assurer sa propre sécurité, comme les habiletés préventives qui concernent l'aménagement et la gestion de dispositifs de « sécurité passive » (dispositifs protecteurs visant à diminuer la gravité d'un éventuel accident : tapis, filet, etc.) qui doivent réellement servir à la récupération d'accidents, simulés ou non, lors d'un apprentissage signifiant.

Par ailleurs, tant que le risque n'est pas maitrisé, il est un obstacle à l'apprentissage. II s'agit alors de veiller à l'adaptation du risque au niveau d'habileté des élèves. L'apprentissage des habiletés sportives, et de façon concomitante des habiletés 


\section{eJRIEPS 35 avril 2015}

d'évitement, nécessite une gradation de la difficulté et une progressivité des facteurs de risque (en modulant par exemple l'incertitude événementielle ou temporelle, la vitesse d'exécution ou la complexité des opérations à réaliser).Chaque élève peut ainsi choisir un niveau de risque adapté et trouver un compromis optimal entre la difficulté de la tâche et la dangerosité du contexte. Ceci présuppose une bonne connaissance de soi pour évaluer ses savoirs et habiletés et la recherche active des modalités de dépassement.

Pour résumer les propositions de Delignières (1991, 1993), l'apprentissage de la gestion du risque en EPS devrait passer par une pratique en situation signifiante, risquée mais adaptée au niveau de l'élève, qui permettra le développement progressif des habiletés spécifiques sportives et leurs indissociables habiletés de sécurité (d'évitement et préventives). Le contexte est cependant différent en école supérieure de cirque: contrairement à I'EPS, la confrontation à des situations risquées signifiantes est incontournable, quotidienne et présente un risque objectif plus élevé, notamment dans les spécialités mêlant l'acrobatique à l'aérien. Par ailleurs, le milieu aseptisé des écoles de cirque est aussi bien différent de la pleine nature au cœur des réflexions de Corneloup et Soulé (2002). Une compréhension de la gestion des risques spécifique aux écoles supérieures de cirque semble alors nécessaire.

2.3. La gestion du risque en école supérieure de cirque : entre apprentissage formel et informel

Si quelques travaux scientifiques se sont intéressés à la question du risque dans le cirque (Fourmaux, 2006 ; Goudart, 2002 ; Sizorn, 2008), pratiquement aucun n'a abordé la question de l'apprentissage de la gestion du risque dans les formations supérieures d'artiste de cirque. L'étude récente de Legendre (2014), qui s'appuie surtout sur des entretiens avec des enseignants des écoles supérieures de cirque en France, met en avant une double transmission de la gestion du risque : une partie de façon formelle et une autre de façon informelle.

La transmission formelle concerne surtout les techniques sportives, mais aussi la gestion de l'intégrité physique ou celle du matériel. L'apprentissage technique se réalise de façon organisée et formelle en s'appuyant sur la maitrise progressive des éducatifs et par la répétition. II est alors possible de prendre de plus en plus de risques dans le sens où il y a une maitrise de plus en plus grande des risques pris. L'entretien physique et l'hygiène de vie sont soulignés également comme contenus formels de formation participant à la gestion du risque (préparation physique notamment), tout comme la maitrise de l'environnement matériel (accroches, parades, tapis, etc.). La transmission informelle 


\section{eJRIEPS 35 avril 2015}

concerne surtout des dimensions psychologisées telles que la maitrise de la peur ou la confiance en soi qui permettraient une gestion du risque. Les enseignants considèrent que la transmission de la confiance en soi, par exemple, est compliquée, et qu'elle va se construire surtout dans les relations aux autres (enseignants et partenaires). Des dimensions plus intra-individuelles sont mises en avant également, comme l'état psychologique de la personne et son rapport au danger auxquels les enseignants essayent d'être attentifs et qu'ils tentent de saisir de manière informelle. Legendre (2014) précise par rapport à ces dimensions : «les causes des blessures (...) mettent en lumière des dimensions personnelles, psychologiques qui vont échapper à la gestion fortement formalisée des apprentissages physiques et sportifs. On aborde ici des dimensions nonformelles qui peinent à être traduites en compétences ou en objectifs d'apprentissage d'un cursus formalisé d'une école supérieure » (p.9).

Le but de la présente recherche est donc d'essayer de mieux comprendre ces dimensions personnelles, psychologiques et la complexité de leur apprentissage. Les théories concernant les déterminants psychologiques de la performance sportive et l'intervention psychologique dans le sport, nous ont alors paru les plus pertinentes comme cadre de référence.

\subsection{Les dimensions personnelles de la performance sportive}

Dans le milieu du sport, beaucoup d'études portent sur les déterminants psychologiques comme éléments de compréhension de la performance sportive. L'intervention psychologique (préparation mentale notamment) s'appuie sur ces données pour optimiser les performances en complément de la préparation physique et de l'entrainement technique et tactique.

Plusieurs dimensions personnelles sont mises en avant dans ces recherches: elles pourront être intra-individuelles (cognitives, conatives, émotionnelles) ou interindividuelles (sociales, émotionnelles). Les dimensions cognitives (Ripoll, 2004) concernent notamment le traitement de l'information, la mémoire, le raisonnement ou l'attention (pour une revue de littérature : Bernier, Thienot \& Codron, 2009 ; Ferrel-Chapus \& Tahej, 2010). Les dimensions conatives se rapportent à la personnalité (Le Scanff \& Nicchi, 2004), aux stratégies de coping (ibid.), aux croyances sur soi (pour une revue de littérature : Famose, Guerin \& Sarrazin, 2005) ou encore aux styles explicatifs (pour une revue de littérature : Martin-Krumm \& Sarrazin, 2004). Les dimensions sociales mettent en avant la cohésion de groupe (pour une revue de littérature : Buton, Fontayne \& Heuzé, 2006), le leadership (Heuzé, 2003), les relations interpersonnelles et compétences relationnelles (Pion \& 


\section{eJRIEPS 35 avril 2015}

Raimbault, 2008), etc. Et enfin, les dimensions émotionnelles concernent en particulier la gestion du stress (Le Scanff \& Famose, 1999), le « flow» ou «l'état psychologique optimal » (pour une revue de littérature: Demontrond \& Gaudreau, 2008), les états affectifs (pour une revue de littérature: Debois, 2003), l'intelligence émotionnelle et la gestion des émotions (Pion \& Raimbault, 2008). Ces dimensions personnelles sont trop nombreuses pour être explicitées et détaillées ici, mais nous reviendrons de façon beaucoup plus approfondie dans la discussion sur celles qui sont en lien avec la gestion du risque des circassiens.

En conclusion de cette revue de littérature, les études sur la gestion du risque en contexte sportif sont rares et celles sur son apprentissage encore plus. II est mis en avant, d'une façon générale, que la gestion du risque se fait en amont, en cours d'action et éventuellement en aval en cas d'accident. II ressort aussi que la gestion du risque et son apprentissage sont évidemment très dépendants du contexte étudié. En école supérieure de cirque, Legendre (2014) montre qu'à côté d'une transmission formelle des techniques sportives et de la gestion de l'intégrité physique et du matériel, la gestion du risque passe aussi par la mise en jeu de dimensions personnelles psychologiques difficiles à formaliser et transmettre. Le but de cette présente étude sera alors d'étudier la gestion du risque des étudiants circassiens à travers la mise en jeu de ces dimensions personnelles et les apprentissages qui en découlent.

\section{Méthodologie}

Cette étude exploratoire qualitative s'appuie sur des entretiens semi-directifs auprès de 30 étudiants en école supérieure de cirque (Ecole Nationale des Arts du Cirque de Rosnysous-Bois et Académie Fratellini à Saint-Denis). Les étudiants, d'une moyenne d'âge de 20,7ans, sont spécialistes de différentes disciplines, plus ou moins aériennes, plus ou moins acrobatiques. Pour préserver l'anonymat des sujets, un numéro leur est attribué. Ce dernier est reporté dans les extraits d'entretiens. 


\section{eJRIEPS 35 avril 2015}

Tableau 1. Caractéristiques et spécialités de la population étudiée

\begin{tabular}{|c|c|c|c|c|c|}
\hline S1 & Femme 20ans & \multirow{4}{*}{$\begin{array}{l}\text { Voltigeur(se) } \\
\text { Bascule Coréenne }\end{array}$} & S16 & Homme 23ans & \multirow{3}{*}{ Mât chinois } \\
\hline $\mathrm{S} 2$ & Homme 22ans & & $\mathrm{S} 17$ & Homme 22ans & \\
\hline S3 & Homme 21ans & & S18 & Homme 18ans & \\
\hline S4 & Homme 22ans & & S19 & Femme 20ans & \multirow[b]{2}{*}{ Tissu } \\
\hline S5 & Femme 21ans & \multirow{3}{*}{$\begin{array}{l}\text { Voltigeur(se) } \\
\text { Main à main }\end{array}$} & $\mathrm{S} 20$ & Homme 23ans & \\
\hline S6 & Femme 18ans & & $\mathrm{S} 21$ & Femme 21ans & Cerceau aérien \\
\hline S7 & Homme 18ans & & S22 & Homme 19ans & Sangle \\
\hline S8 & Femme 18ans & \multirow{2}{*}{$\begin{array}{l}\text { Voltigeuse } \\
\text { cadre aérien }\end{array}$} & $\mathrm{S} 23$ & Homme 25ans & Trapèze Washington \\
\hline S9 & Femme 21ans & & S24 & Femme 23ans & \multirow{2}{*}{$\begin{array}{c}\text { Porteur(se) cadre } \\
\text { aérien }\end{array}$} \\
\hline S10 & Homme 20ans & Fil de fer & S25 & Homme 20ans & \\
\hline S11 & Femme 20ans & \multirow{4}{*}{ Acrobatie } & S26 & Homme 19ans & Porteur main à main \\
\hline S12 & Homme 20ans & & S27 & Homme 23ans & $\begin{array}{l}\text { Jongleur + porteur } \\
\text { main à main }\end{array}$ \\
\hline S13 & Homme 23ans & & $\mathrm{S} 28$ & Homme 19ans & \multirow{2}{*}{ Roue Cyr } \\
\hline S14 & Homme 20ans & & S29 & Homme 24ans & \\
\hline S15 & Femme 21ans & Corde lisse & S30 & Femme 18ans & Equilibre \\
\hline
\end{tabular}

Les entretiens menés auprès des étudiants ont permis de saisir les représentations de leur gestion du risque corporel (notamment les dimensions personnelles mises en jeu) et de l'apprentissage de cette gestion dans leur école respective. La technique de l'entretien est en effet particulièrement adaptée ici puisque, outre le recueil d'informations portant sur des faits (objectifs), elle permet de recueillir des informations qui portent sur des représentations (subjectives) "qui sont tout aussi importantes que les faits eux-mêmes " (De Ketele \& Rogiers, 2009, p.130). Dans ce dernier cas le recueil «vise à recueillir des opinions, des façons de percevoir les choses ou les comportements, à préciser leur signification ou encore à leur attribuer une cause " (ibid., p.125). Les entretiens menés sont semi-directifs car ces derniers paraissent les plus pertinents dans le cadre de cette recherche exploratoire. En effet, les informations recueillies par cette méthode reflètent mieux les représentations que dans un entretien directif car l'interviewé a davantage de liberté dans la façon de s'exprimer (ibid.) et les questions posées, moins directives, n'ont pas pour but de vérifier des hypothèses posées a priori. De plus, les informations recueillies le sont aussi dans un temps beaucoup plus court que dans un entretien libre qui est surtout utilisé pour se forger une hypothèse (ibid.) et qui n'oriente pas les questions. Au contraire, la méthode de l'entretien semi-directif « consiste à faciliter l'expression de l'interviewé en l'orientant vers des thèmes jugés prioritaires pour l'étude tout en lui laissant une certaine autonomie » (Guibert \& Jumel, 1997, p.102). Trois thèmes principaux ont ainsi été retenus, ils composent la base de notre guide d'entretien (Deslauriers, 1991) et seront obligatoirement abordés à travers des questions ouvertes (dont la formulation peut changer) et éventuellement des relances neutres qui permettent de préciser le discours des interviewés. Le premier thème général concerne les représentations du risque des 


\section{eJRIEPS 35 avril 2015}

étudiants dans leur pratique de circassien (e.g., «Raconte-moi, dans tes activités de cirque, est-ce que tu trouves que la prise de risque est importante ? », et relance sur les risques abordés: «C'est-à-dire? C'est quoi «ce » risque? »). Puis dans un second temps est abordée la façon dont ils gèrent ces risques (e.g., « Et tu le gères comment ce risque-là ? »). Enfin le troisième thème concerne l'apprentissage de cette gestion du risque (e.g., «Et d'où tu le tiens ça ? » et relance sur la formation « Et est-ce qu'on vous forme à ça ici ? »).

Les entretiens, intégralement enregistrés et retranscrits mot à mot, ont fait l'objet d'une analyse thématique de contenu de type inductif. En effet, l'étude étant exploratoire, les différents éléments relatifs à la gestion du risque et son apprentissage n'ont pas été définis à l'avance, mais regroupés en thèmes et sous-thèmes a posteriori à partir des données qui ressortaient des discours des étudiants. Par ailleurs, nous ne présenterons dans cet article qu'une partie des nombreuses données recueillies. Tout d'abord, les représentations des différents risques ne seront pas détaillées ici, mais elles ont permis d'amener progressivement la question de la gestion de ces risques par les étudiants et se retrouvent par conséquent en filigrane dans ce deuxième thème d'entretien. Ensuite, seules les dimensions personnelles de la gestion des risques corporels -qui étaient les plus largement évoquées- et leurs apprentissages seront explicités ici, même si les étudiants ont abordé d'autres dimensions notamment matérielles.

Concernant l'analyse thématique de contenu, la thématisation « constitue l'opération centrale de la méthode, à savoir la transposition d'un corpus donné en un certain nombre de thèmes représentatifs du contenu analysé et, en rapport avec l'orientation de recherche » (Paillé \& Mucchielli, 2012, p.232). Pour ce faire, il s'agit de voir comment les différents thèmes, qui émergent d'une lecture entretien par entretien, se recoupent, se complémentent, ou se contredisent : des fusions, des subdivisions, des regroupements et surtout des hiérarchisations thématiques pourront alors être faites (ibid.). Deux thèmes principaux et leurs sous-thèmes respectifs ont ainsi été relevés. II s'agit des dimensions intra-individuelles et des dimensions interindividuelles en jeu dans la gestion du risque corporel. Ces deux thèmes et leurs sous-thèmes seront présentés dans la partie « résultats » avant d'être discutés à la lumière des données scientifiques relatives à la psychologie de la performance sportive. En effet, les thèmes qui émergent de cette analyse sont difficiles à mettre en lien avec les recherches sur le risque, mais sont tous emblématiques des études sur les déterminants de la performance sportive et de l'intervention psychologique dans le sport. Ce cadre d'analyse nous a alors semblé le plus 


\section{eJRIEPS 35 avril 2015}

pertinent et le plus global pour discuter de l'ensemble des dimensions personnelles mises en avant par les étudiants dans leur gestion du risque corporel et son apprentissage. Ce cadre permet également d'avancer quelques pistes d'intervention.

\section{Résultats}

Les résultats obtenus mettent en avant des dimensions intra-individuelles et interindividuelles de la gestion du risque corporel, ainsi que leurs conditions d'apprentissage.

4.1. Dimensions intra-individuelles de la gestion du risque corporel

Trois dimensions intra-individuelles sont mises en avant par les étudiants dans leur gestion du risque : la gestion de la peur, la confiance en soi et en son corps, et la concentration.

4.1.1. La gestion de la peur: un apprentissage informel, un «bricolage » personnel

La donnée la plus importante qui ressort de cette étude exploratoire est le discours quasiunanime de tous les étudiants concernant leur(s) «peur(s) » quand on les interroge sur le risque qu'ils perçoivent dans leur pratique. Ils évoquent surtout la peur de se faire mal, en particulier en acrobatie [« L'acrobatie au sol, bien sûr que j'y vois un risque (...) j'ai très peur! Ce qui me gâche la vie, car j'ai trop peur (...). Alors j'ai peur de quoi ? J'ai peur de quoi ? C'est con quoi, mais j'ai peur de tomber, j'ai peur de me faire mal, mais de me faire mal violemment, de me rétamer, de me fracasser au sol » (S11, acro-danse)] ou le vertige pour les disciplines aériennes [«Au mât, sur ma spé, je suis plutôt calme, je fais attention parce que ça me fait peur, parce que j'ai le vertige donc... j'ai une part qui me freine parce que j'ai peur » (S16, mât chinois)]. Des étudiants font cependant la différence entre la «bonne » peur, qui donne envie de s'engager et qui est surtout liée à la recherche de sensations ["Je sais que j'aurai peur mais c'est une peur que j'ai envie d'avoir quoi, comme faire de là-haut du trapèze volant, faire des saltos tout ça! Voilà recherche de sensations » (S1, voltigeuse bascule coréenne)] et la mauvaise peur qui tétanise. La peur serait en effet très handicapante pour la plupart d'entre eux, entrainant un « blocage » que les élèves ont du mal à raisonner: ils n'arrivent pas à lancer le mouvement [«Mon cerveau, il bloque et il m'empêche de faire le mouvement, euh par peur, par appréhension réelle, alors que j'ai visualisé le mouvement, j'ai envie de le faire, mais euh, mais là y'a quelque chose en moi qui me bloque et euh qui dit "non n'y va pas !" » (S17, mât chinois)]. Cette peur pourrait engendrer un risque accru d'accidents notamment à cause du 


\section{eJRIEPS 35 avril 2015}

« refus » de certaines figures, c'est-à-dire en stoppant la figure au milieu du mouvement ce qui entrainerait une réception aléatoire et dangereuse [«Quand on a peur on prend des positions débiles et du coup l'autre n'arrive plus à nous rattraper ou on se bloque » (S5, voltigeuse main à main) ; «Si tu fais une figure, tu le fais en entier quoi ! (...) II vaut mieux faire la figure et pas bien la faire, que ne pas la faire et de commencer à la faire parce que la tête passe en premier et là on tombe la tête la première et c'est là que ça peut faire mal ! » (S7, voltigeur main à main)].

Pour les étudiants, la peur serait donc un des facteurs de risque les plus importants, ce qui présuppose alors que leur gestion du risque passe en premier lieu par une gestion de leur peur. Les étudiants précisent que cette gestion de la peur serait éminemment personnelle [ «Bon, c'est quand même très personnel, la gestion de la peur (...) c'est dans la tête, je veux dire » (S12, acrobatie), “ On ne t'apprend pas à... à gérer tes peurs, mais parce que je pense qu'au final, on ne peut pas vraiment te l'apprendre, c'est quelque chose que tu trouves tout seul » (S17, mat chinois)].

Par conséquent, pour la plupart des étudiants, la gestion de la peur et donc du risque lié à cette peur, passerait principalement par des techniques informelles et personnelles, issues notamment de l'expérience. Selon les étudiants, ces techniques ne feraient pas l'objet d'un apprentissage formel particulier, et trouver «sa " technique poserait de toute évidence des difficultés [ "Je me bats chaque jour pour vaincre ma peur mais ce n'est pas évident» (S11, acro-danse), “Pff je ne l'ai pas encore vraiment appris hein! Je l'expérimente, parce que ça bloque encore toujours » (S17, mat chinois)].

Les étudiants gèreraient donc leur peur de façon autonome avec deux techniques en particulier : « se lancer sans réfléchir » et/ou se rassurer sur ses capacités avant de se lancer ["Y'a toujours la peur, mais on sait faire le mouvement et c'est là que ma technique de se dire "je sais faire ce mouvement, je peux le peux faire", éteindre son cerveau et $y$ aller, là elle marche » (S12, acrobatie)]. Pour certains étudiants, « ne pas réfléchir » signifie ne pas penser aux conséquences d'un mouvement raté [«Tu dois arrêter de penser aux conséquences (...) hypothèse s'il y a erreur qu'est-ce qu'il va se passer? Nuque tordue, cassée ? » (S17, mat chinois)] ou s'en remettre à l'automaticité des mouvements maitrisés [ "Il faut vraiment arriver à se vider l'esprit, euh un minimum avant de faire notre figure. Moi, je vois ça quand je suis... quand je suis très fatigué par exemple, ça peut m'arriver de bien réussir, parce que je fais ça machinalement, c'est... sans réfléchir. Pas pour les mouvements trop compliqués, parce que là je ne peux pas » (S12, acrobatie)]. 


\section{eJRIEPS 35 avril 2015}

Des techniques plus classiques de gestion de la peur, comme l'utilisation de la respiration ou la visualisation, sont très peu évoquées par les étudiants.

4.1.2. La connaissance et la confiance en soi et en son corps consécutifs à un apprentissage technique progressif

Un thème récurrent dans la gestion du risque des étudiants concerne le fait de se connaitre, connaitre ses limites, se faire confiance afin de contrôler et d'avoir un risque mesuré [ « Le risque c'est de ne pas avoir confiance en soi, je pense (...) Ben oui, parce que si on sait ce qu'on fait, enfin... y'a pas de risque » (S24, porteuse cadre aérien)].

Cependant cette connaissance ou confiance en soi n'est pas forcément conscientisée. Elle passe souvent par un ressenti, une intuition, un «feeling », pour savoir si on est prêt ou pas. Beaucoup d'étudiants utilisent le terme de le « sentir » ou pas [ "Si je le sens j'y vais, si je le sens pas, j'y vais pas. Je ne me pose pas la question! » (S4, voltigeur bascule coréenne), «Enfin, encore ça dépend vraiment de mon état d'esprit, de comment je me sens sur le moment, ça marche... enfin je marche beaucoup en ressenti (...) pour tout ce que je fais ouais, pour $90 \%$ des trucs que je fais, je marche au feeling quoi, c'est si je le sens. C'est quelque chose d'un peu inexplicable » (S26, porteur main à main)].

La connaissance de son corps dans l'espace, la confiance dans les sensations de son corps et dans ce que « sait faire » son corps est également largement mise en avant dans la gestion du risque des étudiants qui évoquent la technique corporelle et les « réchappes» [ «Moi quand je me lance dans quelque chose, je sais que je vais à peu près retomber sur mes pieds (...) On nous apprend à connaître notre corps et à connaître notre corps dans l'espace. Du coup, si tu sais où tu es en l'air, t'as peu de chance de tomber sur la tête» (S4, voltigeur bascule coréenne)]. En effet, une figure réalisée parfaitement n'est pas accidentogène et en cas de problème, les étudiants ont développé un repérage dans l'espace qui leur permet de tomber avec le moins de dommages possibles (« réchappes») [«On finit par devenir un peu des chats quoi, comment essayer de se retrouver sur ses pieds quand on est mal parti, comment essayer de finir quand même la figure, ou de sentir que ça va pas du tout le faire. Donc il faut tout faire pour essayer, dans tous les cas, de se retrouver sur ses pieds, ou se retrouver dans une position pour pouvoir amortir le choc » (S2, voltigeur bascule coréenne)].

Cette connaissance et cette confiance dans son corps proviennent d'un travail quotidien et de l'expérience, notamment après des chutes [ "C'est pas quelque chose de magique ! C'est un travail de tous les jours, comme les médecins, comme les banquiers. Et alors c'est ça, après tu connais ton corps, tu connais où tu te trouves. Alors, même si pour les 


\section{eJRIEPS 35 avril 2015}

personnes tu es fou, tu sais ce que tu es en train de faire ! » (S23, trapèze Washington), « J'ai pris des repères aussi, que je n'avais pas avant (...) maintenant j'ai pris confiance dans mon corps, dans la manière de tomber (...) mais ça par exemple, ça s'apprend au fur et à mesure quoi, en faisant. (...) après y'a des trucs, tu t'en rends bien compte tout seul, quand tu te prends une boite [rires] ! (S9, voltigeuse cadre aérien)].

Finalement les étudiants sont unanimes pour dire que ce sont les éducatifs, l'apprentissage par étapes, avec du matériel adapté notamment (sécurité passive), et les répétitions qui permettent cette connaissance et cette confiance en soi et en son corps [ "Puis y’a des étapes aussi dans une figure, tu ne vas pas lancer la figure, passer du rien au tout (...) tu as plein d'éducatifs pour t'amener à la figure finale, du coup tu prends des risques modérés. Petits risques par petits risques au lieu de faire un gros risque d'un coup» (S4, voltigeur bascule aérienne), « [Pour gérer la peur] peut être que le travail ça passe par les éducatifs, par exemple pour un mouvement, ça passe par des éducatifs, après ça passe par la longe si y'a besoin de longe, après ça passe par des gros tapis d'abord, puis on réduit l'épaisseur des tapis, jusqu'à arriver au sol (...) C'est faire, faire, refaire encore, jusqu'à comprendre des trucs et se faire confiance en fait » (S10, fil de fer)]. L'apprentissage formel de la technique, et la maitrise sont donc largement au service de la gestion du risque et de la peur

\subsubsection{La concentration}

Le thème de la concentration est un peu moins mis en avant par les étudiants, mais il reste cependant important, en particulier dans les disciplines les plus dangereuses [« Je ne ressens pas les mêmes choses quoi, je suis plus en attention quand je suis sur un trampoline ou quand je pense à faire des choses dangereuses. Je tombe, ben, et bien c'est la mort quoi, tu peux mourir quoi ! Là, je change plus, je ne suis pas pareil, je me mets plus en état où il faut être hyper concentré quoi (...) en général c'est quand t'es pas concentré que tu te blesses » (S14, acrobatie)]. Des étudiants expliquent cette nécessité de ne penser à rien d'autre qu'à sa prestation [ "Dès qu'il y a des éléments un peu parasites dans la tête qui nous font un peu lâcher la concentration, là, ça devient dangereux. Il faut être complètement à fond dans ce qu'on fait et ne pas penser à autre chose à un moment, sinon ça ne marche plus quoi. Quand on fait quelque chose, c'est que ça, et jusqu'au bout » (S2, voltigeur bascule coréenne), «Si je tombe, je suis mort, voilà ! (...) Si je suis en train de penser à la situation de ma famille (...), que mon père n'est pas bien ou... il faut laisser ça, dans les moments où je monte parce que ça, ça n'a rien à voir. C'est la concentration ! C'est comme la méditation euh ça ne veut pas dire que 


\section{eJRIEPS 35 avril 2015}

je ne dois pas penser. Je dois être dans ce que je suis en train de faire » (S23, trapèze Washington)].

\subsection{Dimensions interpersonnelles de la gestion du risque corporel}

4.2.1. La gestion de la peur et du risque : la confiance en l'enseignant

La confiance en l'enseignant est également un thème récurrent du discours des étudiants. Ces derniers s'engageront d'autant plus facilement dans des mouvements dangereux ou difficiles si les enseignants les y encouragent. Cependant, une forte dépendance vis-à-vis de leur enseignant émerge parfois [ "Mais je me sens pas capable de faire un truc super dangereux si mon prof il me dit pas "vas-y tu peux le faire !" (...) Je sais que mes profs, là, je leur fais confiance, donc je sais que si ils me disent que je peux le faire, c'est juste que je peux le faire, donc je vais le faire »(S26, porteur main à main), « J'avais entièrement confiance en lui et je ne me suis jamais fait mal en trampo (...) j'ai réussi à faire des trucs bien quoi, que je n'arrive plus à faire maintenant, parce qu'il n'est plus là non plus (...) Ouais et cette confiance là je l'ai... enfin, après ça ne vient pas non plus comme ça d'un coup, mais... mais ouais, ça me permet d'oublier complètement, ce risque-là, et puis même tous les risques en général d'ailleurs » (S25, porteur cadre aérien)]. Dans cette relation de confiance, de dépendance, un étudiant évoque même son déplacement de responsabilité envers l'enseignant ["J'avais l'impression de déplacer un peu ma responsabilité sur lui. Dans le sens, si lui te fais confiance, il te dit "fais ce mouvement", tu peux le faire, il te l'a expliqué... II te dit "maintenant fais-le !", ben c'est comme si, "je le fais, mais si je me fais mal, de toute façon, ça ne sera pas de ma faute "donc je ne peux pas me faire mal (...). Si je n'ai pas confiance en moi pour le faire, parce que j'ai peur de moi-même, de ne pas réussir à faire ce mouvement, lui il a confiance en moi donc c'est bon, c'est sa responsabilité presque » (S17, mat chinois)].

Cette confiance très importante dans les enseignants vient notamment de la connaissance très fine qu'ils ont de leurs étudiants [ "Ils [les profs] disent ça tu peux envoyer, ça tu ne peux pas envoyer, euh... ça tu vas attendre un peu, t'es pas prêt (...) souvent ils voient juste » (S4, voltigeur bascule coréenne), « En fait, il faut que le prof, il soit là aussi pour te pousser jusqu'au bout de tes limites mais sans te briser, sans te casser. II faut toujours qu'il soit sur le fil de la... de la blessure, ou de... je ne sais pas... du craquage quoi. Mais sans te faire craquer, sans te blesser quoi ! » (S18, mat chinois)].

4.2.2. La gestion de la peur et du risque : la confiance en son/ses partenaire(s) Si les étudiants ont une confiance très importante en leur enseignant, celle-ci est également incontournable à l'égard de leur(s) partenaire(s). En effet, parmi les activités 


\section{eJRIEPS 35 avril 2015}

circassiennes des étudiants interrogés certaines se pratiquent en duo (le main à main et le cadre aérien qui comportent un voltigeur et un porteur) ou en collectif (la bascule coréenne où tous sont voltigeurs, mais assurent aussi le rôle de pareurs). Dans ces disciplines, l'intégrité physique des voltigeurs est entre les mains du porteur. Les étudiants qui pratiquent avec des partenaires mettent tous en avant l'importance de la confiance qui existe déjà entre eux ou qui se crée [«Dans le main à main, en fait c'est important, cette confiance avec l'autre (...) Mon premier partenaire, j'étais tellement en confiance avec lui et j'ai grandi avec lui (...) je ne me suis jamais posé la question en fait... je savais qu'il me rattraperait, que je pouvais faire n'importe quoi (...). [Avec le nouveau porteur] ça fait beaucoup plus peur mais je pense que je m'en remets plus à moi actuellement qu'à lui et me dire que lui il fait son job, que moi je fais mon job, et que du coup cette confiance elle se créera petit à petit (...) Du coup c'est ça qui fait que j'aurai moins peur et que je prendrai moins de risque» (S5, voltigeuse main à main)]. En collectif de bascule coréenne, certains voltigeurs se connaissent depuis de nombreuses années, ce qui favorise cette confiance en l'autre de façon générale et dans l'activité de façon spécifique, avec une fois de plus le risque de dépendance à l'autre [«Et puis la bascule, ça ne se pratique pas tout seul, et moi, je voulais un truc de groupe. On a un collectif, on est déjà des amis à la base... Et du coup, oui voilà, je voulais un truc vraiment de confiance où il y ait tout le monde qui soit présent et pas un agrès tout seul. Ce qui est un peu le problème maintenant, c'est qu'on est un peu dépendant des autres. Mais, c'est ça que je voulais à la base aussi. C'est vraiment avoir une place dans un collectif et de se plaire en dépendant un peu des autres et que les autres dépendent de moi, quoi. J'aime bien cette idée » (S2, voltigeur bascule coréenne)].

\subsubsection{La gestion de la peur et du risque : l'émulation du groupe}

L'émulation de groupe permet aussi de dépasser sa peur et de se lancer dans des figures dangereuses [ «A chaque fois j'avais peur de le faire mais je le faisais quand même. II me disait : "allez !" (...) On regarde les autres, on se dit il le fait (...), on s'évalue, on se dit : "j'suis capable de faire la même chose ! Si lui il y arrive, il y a pas de... enfin, il n'a pas des capacités extraordinaires par rapport à moi. Je peux le faire aussi ! " » (S7, voltigeur main à main), « Après y'a une autre façon aussi d'enlever les risques... enfin d'enlever, non pas d'enlever le risque, le risque y est toujours, mais de gérer le risque, d'enlever un peu la peur, c'est l'adrénaline et le mouvement de groupe en fait. Quand on est plusieurs, souvent on est poussé par une énergie et ça permet (...) d'y arriver, ouais d'y arriver euh 


\section{eJRIEPS 35 avril 2015}

parce qu'on... parce qu'on réfléchit moins (...) et si on pense à trop de trucs, on ne peut pas faire le mouvement, c'est impossible » (S12, acrobatie)].

\section{Discussion des dimensions personnelles de la gestion du risque et de leur apprentissage}

Les données scientifiques issues de la psychologie du sport nous ont semblé les plus pertinentes pour analyser les résultats obtenus: le champ des déterminants psychologiques de la performance pour discuter de la gestion du risque et le champ de l'intervention auprès du sportif, en particulier la préparation mentale, pour discuter de l'apprentissage de cette gestion du risque et proposer quelques pistes de formation.

Dans une première partie, nous nous intéresserons plus précisément aux dimensions intra-individuelles et dans une deuxième partie, aux dimensions interindividuelles.

5.1. Les dimensions intra-individuelles de la gestion du risque corporel

La gestion du risque passerait tout d'abord par la gestion de la peur pour la majorité des étudiants, voire pour la totalité de ceux qui pratiquent les disciplines les plus risquées (avec des éléments acrobatiques et/ou aériens). En plus de la gestion de la peur, deux autres thèmes reviennent majoritairement dans le discours des étudiants: la connaissance et la confiance en soi et en son corps, consécutives à un apprentissage technique progressif, et dans une moindre mesure la nécessité de la concentration. Pour mieux comprendre l'importance de ces trois thèmes dans la formation des étudiants circassiens, nous allons les mettre en lien avec les théories et les techniques conceptualisées en psychologie du sport.

En préparation mentale, la gestion de la peur par exemple, est abordée sous la forme de la gestion du stress. Cette dernière, chez les sportifs, est souvent nécessaire pour faire face aux enjeux de la compétition. Cependant, la gestion du stress du sportif est spécifique. Le Scanff $(1999,2003)$ explique quelles sont les différentes stratégies qui peuvent être employées pour gérer le stress dans le sport de haut niveau, mais aussi dans les situations dites « extrêmes » (armée, missions spéciales, etc.) où la prise de risque est également un élément important. Quatre types de stratégies permettent de gérer le stress et seront à travailler plus ou moins conjointement selon la situation : développer son sentiment de contrôle sur l'environnement, développer un apprentissage technique poussé, développer les techniques individuelles de gestion du stress et développer les relations interpersonnelles du groupe. Nous allons approfondir tous ces thèmes qui sont 


\section{eJRIEPS 35 avril 2015}

également les plus abordés par les étudiants quand ils parlent de leur gestion de la peur et du risque.

1-Développer son sentiment de contrôle sur l'environnement par une meilleure connaissance de celui-ci, l'incertitude déclenchant du stress (Le Scanff, 1999, 2003). Chez les circassiens, la diminution de l'incertitude de l'environnement passe essentiellement par les vérifications matérielles et les protections (accroches pour les disciplines aériennes, tapis, longe, etc.). Ce thème de la « sécurité passive » ne fait pas l'objet de cette étude, mais de façon générale, les étudiants se disent bien sensibilisés sur ce sujet avec, dans une des deux écoles, des apprentissages formels lors de cours théoriques (« On a eu des formations plus techniques sur comment accrocher une sangle, et tout») et des apprentissages informels quotidiens lors des entrainements (« [Le prof] a dit: "alors là vous ne mettez pas de tapis parce que y'a pas de risque, c'est sûr à $100 \%$ qu'il va atterrir sur ses pieds ?" Mais alors là, à ce moment-là, tu vas mettre un tapis »).

2-Développer un apprentissage technique poussé pour éviter la dégradation de performance due au stress. Un surapprentissage (entrainement intensif) en condition réelle permet notamment aux tâches de devenir automatiques et de requérir moins d'attention augmentant ainsi le sens du contrôle c'est-à-dire l'impression de pouvoir contrôler la situation (Le Scanff, 1999, 2003). Les étudiants circassiens mettent largement en avant la dimension «technique » dans leur gestion du risque et de la peur. L'apprentissage technique progressif et les répétitions permettent l'automaticité de certains mouvements (« [Le professeur] va te faire faire plein d'éducatifs pour que ton corps, il enregistre les mouvements ») et la connaissance du corps dans l'espace («Tu tournes ton corps dans tous les sens et au bout d'un moment tu sais où il est quoi que tu fasses »). Cette dernière est primordiale, tant pour les techniques corporelles que pour les « réchappes». Ces «tactiques d'urgence » sont des habiletés d'évitement (Delignières, 1993) qui permettent de retomber sans se faire mal quand une figure est mal exécutée («On finit par devenir un peu des chats »).

Par ailleurs, l'apprentissage technique progressif et répétitif en favorisant la maitrise, augmenterait aussi la connaissance et la confiance en soi et dans son corps, ce qui permettrait une diminution de la peur et des risques de façon générale ( $P l u s$ t'es fort, plus t'as confiance en toi et du coup moins t'as peur »). Le Scanff (1999, 2003) confirme qu'une meilleure maitrise des habiletés augmente le sentiment d'efficacité personnelle qui réduit alors le stress. Ce sentiment d'efficacité personnelle (attente de réussite dans une situation très particulière) dépend en effet des performances récentes dans la tâche 


\section{eJRIEPS 35 avril 2015}

(Bandura, 1977). Cependant si les sportifs se sentent efficaces, confiants dans la tâche parce qu'ils sont performants, ils sont aussi performants parce qu'ils se sentent confiants (e.g., Gould, Weiss \& Weinberg, 1981): confiance et efficacité sont donc interdépendantes. Pour les étudiants qui n’ont pas confiance en eux (« Et je pense que je n'ai pas confiance en moi et c'est très dur ça aussi, parce que je pense que ça m'aiderait »), un travail sur la confiance pourrait les aider à se lancer dans des figures difficiles, la réussite nourrissant en retour leur confiance. Des techniques spécifiques existent notamment en préparation mentale.

3-Développer les techniques individuelles de gestion du stress. Le Scanff, (1999, 2003) indique que la majorité des techniques utilisées (relaxation, méditation, etc.) vise une diminution des processus physiologiques de l'activation déclenchés en condition de stress (baisser l'anxiété somatique qui se manifeste par une accélération du rythme cardiaque, des tensions musculaires, etc.). Cependant, certaines études montrent que la réduction de l'activation n'est pas toujours souhaitable en vue d'une performance sportive. Les étudiants circassiens évoquent aussi la « bonne peur », cette recherche de sensations qui favorise l'engagement dans l'action. II est donc parfois préférable de privilégier des techniques qui diminuent l'anxiété cognitive (expectations pessimistes, manque de concentration, etc), et non l'anxiété somatique, comme l'imagerie mentale ou le dialogue interne qui modifie l'évaluation des situations stressantes. Certaines techniques composites comme le SIT (stress inoculation training, Meichenbaum, 1977) ont l'avantage de traiter en même temps l'anxiété somatique et cognitive et d'incorporer les stresseurs dans l'entrainement. D'autres techniques reposent sur l'idée que pour certaines performances, ce sont les secondes qui précèdent l'habileté motrice qui sont déterminantes (Le Scanff, 1999, 2003). En effet, dans les sports à habiletés fermées très automatisées, l'anxiété augmente l'attention portée sur le geste, et ce passage d'un contrôle inconscient à un contrôle conscient déstabilise les acquisitions (Masters, 1992). Dans ces disciplines spécifiques (sports de précision, plongeon, gymnastique, etc.), il faut alors éviter cette désautomatisation. Dans ce cas, l'utilisation de «routines de performance » pour gérer le stress et amener le sportif à un «état de performance optimal " a démontré son efficacité. Ces routines sont un ensemble de schémas de pensées, d'actions ou d'images que l'on reproduit systématiquement avant d'exécuter une performance (Crews \& Boutcher, 1986). Elles permettent de contrôler et de diriger ses émotions, ses pensées et son attention juste avant une performance pour basculer dans l'automatisme (Singer, 2002). Par ailleurs, c'est à chacun de trouver sa propre recette en 


\section{eJRIEPS 35 avril 2015}

utilisant les stratégies ou habiletés mentales de base les plus adaptées à ses caractéristiques personnelles (Gould \& Udry, 1994), à sa discipline et son niveau de pratique. Ces stratégies concernent surtout le domaine de la concentration et consistent en l'utilisation du dialogue interne (discours lié à l'exécution technique, des encouragements) ou de l'autosuggestion (se persuader qu'on y arrivera), de l'imagerie mentale (visualisation d'un mouvement), des contrôles respiratoires, et de la relaxation pour diminuer l'activation ou, au contraire, de la mise en tension pour l'augmenter (Le Scanff, 1999, 2003).

Dans les écoles supérieures de cirque, les étudiants ne disposent pas de préparateur mental ou de psychologue du sport pour leur apprendre ces techniques individuelles de gestion du stress. Comme dans d'autres disciplines sportives où la préparation mentale, même à haut niveau, n'est pas utilisée pour diverses raisons, les athlètes ont donc plutôt tendance à «bricoler» leurs propres techniques. Ripoll (2008) qui a interviewé des sportifs de haut niveau a été surpris de leur capacité à faire face seuls aux moments difficiles notamment, sans aucune préparation mentale. Ils font à « leur propre sauce... et ça marche » (ibid., p.219). En plus d'avoir une très grande confiance en eux, ils sont passés maitre dans la gestion de leur mental sans intervention extérieure : gestion du stress, stratégies d'autorenforcement, etc. Les étudiants circassiens «bricolent » aussi leurs techniques de gestion de la peur et du risque ( "Je ne l'ai pas encore vraiment appris hein, je l'expérimente, parce que ça bloque encore toujours ») et semblent utiliser avant l'exécution d'une figure dangereuse des techniques qui s'apparentent aux routines de performance. La réalisation de figures acrobatiques complexes, précises et risquées en cirque (bascule coréenne, disciplines aériennes, etc.) s'intègre bien dans la catégorie des habiletés fermées où la précision est importante et pour laquelle les routines de performance, qui privilégient concentration et automaticité du mouvement, sont adaptées et efficaces. La technique décrite par la très grande majorité des étudiants pour gérer leur peur semble peu académique, mais efficace : ne pas réfléchir et se lancer. Certains étudiants expliquent l'importance de ne pas trop réfléchir pour ne pas penser aux conséquences possibles d'un mouvement raté avant de partir («Si je réfléchis trop, c'est foutu... je me vois, je visualise le mouvement, je me vois me scratcher »). Des termes forts sont employés par les étudiants pour parler de cet état de «non-réflexion » : « mettre mon cerveau sur off », « déconnexion de la conscience », « se vider l'esprit », « éteindre son cerveau ". Même si cela n'est pas conscientisé chez la plupart, il semblerait que cette stratégie permette également d'éviter la perturbation du mouvement une fois celui-ci 


\section{eJRIEPS 35 avril 2015}

automatisé. La majorité des figures exécutées par les circassiens atteignent, après apprentissage, un stade de traitement automatique qu'un contrôle conscient dégraderait. Les comportements automatiques sont inconscients, rapides, ne nécessitant pas de stratégies cognitives particulières (Shiffrin \& Schneider, 1977), et se développent avec l'entrainement, la répétition dans des conditions similaires. De façon générale, il serait préférable de centrer son attention sur les effets du mouvement plutôt que sur le mouvement lui-même une fois qu'il est automatisé (Wulf \& Prinz, 2001) ou d'utiliser une focalisation attentionnelle externe (Ferrel-Chapus \& Tahej, 2010) comme la cible au golf par exemple (Crews \& Landers, 1991). Une voltigeuse en main à main dit à ce sujet: « J'essaye de mettre mon cerveau sur off. De me remettre à ce que mon corps connait et puis lui faire confiance en fait », " Je ne fais que penser par exemple... à l'endroit où doit passer mes jambes, plutôt qu'à l'ensemble en fait. J'adore analyser tout, tout ce que je fais, voir plus large et tout, mais je ne m'en sors pas. Du coup, je me cale sur un point, l'endroit où doit passer mes jambes et puis je sais que ça fonctionnera... Je ne pense pas au mouvement en lui-même, je pense à un truc, du genre mes pieds, voilà ! Et du coup, toute cette peur qui parasite autour parce que je pense que je dois tirer mes bras, parce que je pense que je dois me mettre en boule, parce que je dois m'ouvrir à ce moment-là, tout ce qui me fait peur du coup, parce que j'ai peur de ne pas le faire au bon moment, je l'efface et je me concentre sur un truc que je sais exactement ». Par ailleurs, s'ils essayent de ne pas réfléchir aux conséquences d'une erreur, la plupart des élèves se rassurent aussi brièvement sur leur capacité avant de se lancer dans le mouvement. Cette technique bien connue en préparation mentale relève également des routines de performance : le « dialogue interne » voire l'autosuggestion (Bandura, 1977) (« Je me dis "la technique tu la connais, [le prof] il te l'a apprise, et voilà tu sais la faire" et j'y vais ! »).

Concernant des techniques un peu plus spécifiques, très peu d'élèves évoquent l'importance de la respiration avant de faire un mouvement difficile ou de l'imagerie mentale, alors que cette dernière technique est couramment utilisée, même de façon informelle, par les sportifs. Elle semblerait pourtant très pertinente pour visualiser les mouvements complexes à réaliser, ce qui favoriserait l'apprentissage, la concentration et la gestion du stress (Perreaut-Pierre, 2000).

Finalement en ce qui concerne les dimensions intra-individuelles, la gestion de la peur et du risque par les étudiants circassiens repose sur un apprentissage formel de techniques corporelles et un apprentissage informel de techniques mentales. L'apprentissage des 


\section{eJRIEPS 35 avril 2015}

techniques corporelles favorise la maitrise voire l'automatisation de certains mouvements (gestes ou réchappes) et la confiance en soi et en son corps. Les techniques mentales favorisent un état de concentration (dialogue interne notamment) et le déroulement automatique du mouvement (ne pas penser au mouvement pour ne pas le perturber). Ces « routines de performance » semblent pertinentes au regard des données concernant la gestion du stress. Néanmoins, à l'instar de Ripoll (2008) qui se demande si les sportifs de haut-niveau qu'il a interrogés n'auraient pas fait encore une plus belle carrière en ayant une préparation mentale dispensée par des professionnels plutôt qu'en se la construisant tout seul au fil des expériences, nous pouvons nous demander si des interventions spécifiques en préparation mentale (gestion du stress) ne seraient pas intéressantes, tant la gestion de la peur est un sujet de préoccupation majeur de la plupart des étudiants. II semble notamment que l'imagerie mentale ou certaines techniques de respiration seraient pertinentes dans ce cadre.

4-Développer la gestion des ressources d'une équipe, en particulier le développement des relations interpersonnelles, permet un soutien social qui est une des stratégies les plus efficaces pour gérer le stress (Le Scanff, 1999, 2003). L'importance des dimensions interpersonnelles a aussi largement été abordée par les étudiants circassiens dans la gestion du risque et va faire l'objet de la deuxième partie de cette discussion.

5.2. Les dimensions interindividuelles de la gestion du risque corporel

Les dimensions interindividuelles de la gestion de la peur et du risque ont été fortement mentionnées par les étudiants à travers les relations et la confiance qu'ils ont en leur entraineur mais aussi en leurs partenaires.

5.2.1. Relations avec l'entraineur : une confiance à toute épreuve

La relation entraineur-entrainé est un élément indissociable de la pratique sportive en club et semble être un déterminant incontournable de la performance sportive, pour autant les chercheurs ne se sont intéressés que récemment à cette relation. Les premières recherches sont celles concernant le leadership. Elles ont surtout étudié l'entraineur à travers l'étude de ses caractéristiques intra-personnelles : ses comportements, ses prises de décisions et ses connaissances (Heuzé, 2003). Toutefois, ces études ne prennent pas en compte le versant affectif, émotionnel qui offrirait pourtant un contexte favorable à l'entrainement et à la performance (Lévêque, 1995 ; Totchilova-Gallois, 2005). Quelques recherches d'origine anglo-saxonnes récentes se sont intéressées de façon spécifique aux relations interpersonnelles entre l'entraineur et le sportif. Losier et Vallerand (1995) ont montré que l'entraineur, notamment, devait essayer de développer avec ses athlètes une 


\section{eJRIEPS 35 avril 2015}

relation harmonieuse, valorisante, satisfaisante et qui amène les sportifs à lui faire confiance. Jowett et Meek (2000) définissent les relations interpersonnelles entraineursportif comme une interaction entre un ensemble d'émotions («proximité » affective, confiance et respect mutuels), de pensées («co-orientation » avec un cadre commun à trouver nécessitant du dialogue, des négociations, des prises de décisions) et de comportements («complémentarité » basée sur la collaboration, le travail d'équipe entre l'entraineur et le sportif). Pour Saury (2004) cependant, il faudrait remettre en question les deux pôles distincts de la relation entraineur-entrainé : l'un « relationnel ou affectif » et l'autre « instructif » (faire acquérir des procédures techniques et tactiques), ainsi que la conception selon laquelle l'entraineur prescrit et le sportif exécute. Des recherches ont, en effet, mis en avant la véritable collaboration organisée en vue d'un travail collectif, il faudrait alors plutôt parler de « collaboration entraineur-athlètes ». Cette dernière s'appuie notamment sur un référentiel commun, fruit d'une expérience partagée (Saury \& Durand, 1995) prenant en compte l'évocation des sensations de sportif par l'entraineur, une connaissance fine des sportifs et l'évocation d'anecdotes passées vécues en commun.

Les données qui ressortent des entretiens avec les étudiants circassiens concernant leur gestion du risque mettent en avant les deux pôles classiques de la relation entraineursportif : l'un technique et l'autre affectif. Ces deux pôles sont cependant interdépendants et largement basés sur la confiance en l'entraineur. Le pôle technique, a déjà été mentionné : les étudiants gèrent de mieux en mieux la peur et les risques à mesure que leur maitrise augmente, en particulier la maitrise de leur corps dans l'espace et l'automatisation des mouvements. Or, cette maitrise passe par les répétitions mais aussi par les éducatifs et diverses situations d'apprentissage mises en place par les enseignants. Les étudiants font entièrement confiance aux enseignants, qui sont des experts dans leur discipline, pour ces apprentissages techniques. Aucun étudiant n'a mentionné quelques désaccords d'ordre technique concernant l'enseignement reçu. La relation de confiance est surtout mise en avant, et de façon unanime par les étudiants, au niveau des encouragements pour se lancer dans des figures difficiles voire dangereuses ( "Dans ce cas-là je lui fais confiance... s'il dit que je peux le faire, je le fais »). Cette confiance en l'enseignant (pôle affectif) provient donc de son expertise technique (pôle technique) qui rassure les étudiants, mais aussi de la connaissance précise qu'il a du fonctionnement de ses étudiants. Cette dernière lui permet de s'adapter à leurs attentes et caractéristiques ( Quand elle sent que je suis fatigué, elle va faire un travail avec mon état aussi parce que ça sert à rien de bosser des trucs alors que tu n'en es pas capable 


\section{eJRIEPS 35 avril 2015}

»). Saury et Durand (1995) ont d'ailleurs montré que les entraineurs évoquent une connaissance très fine des sportifs pour interpréter les comportements de ces derniers et pour justifier des modes d'intervention différenciés à leur égard. C'est un des éléments du « référentiel commun » partagé avec l'athlète qui constitue une des bases de la coopération entraineur-sportif.

La confiance en l'entraineur est cependant un thème très peu abordé dans les études sur les relations entraineur-sportif. II s'avère que dans la gestion spécifique de la peur et du risque, cette confiance est un élément pourtant primordial qui mériterait un regard plus approfondi. Nous allons maintenant aborder la confiance dans les partenaires qui est également largement mise en avant par les étudiants.

\subsubsection{Relations avec les partenaires : la confiance et l'émulation de groupe}

Les relations interindividuelles dans un groupe sportif sont surtout étudiées à travers le thème de la cohésion (Buton, Fontayne \& Heuzé, 2006 ; Heuzé, 2003). Si de nombreuses conceptions théoriques de la cohésion existent, les recherches font clairement ressortir deux dimensions (Cox, 2005). La cohésion opératoire face à l'exécution des tâches, qui est le degré de collaboration d'une équipe dans la poursuite d'un but opératoire, et la cohésion sociale qui est le degré d'attirance entre les équipiers et leur degré de satisfaction à évoluer ensemble. Dans le domaine sportif, il semble qu'un consensus se dégage autour du modèle de Carron, Widmeyer et Brawley (1985) en quatre facteurs (intégration opératoire du groupe, attractions individuelles opératoires pour le groupe, intégration sociale du groupe, attractions individuelles sociales pour le groupe). La cohésion est donc décrite comme une variable multidimensionnelle mais aussi dynamique: elle fluctue rapidement et ne s'exprime pas forcément par ces quatre facteurs. Les recherches ont surtout mis en avant les déterminants et les conséquences de la cohésion, et notamment son lien avec la performance sportive. Quelques résultats montrent également que les relations interpersonnelles permettent un soutien social qui est une des stratégies les plus efficaces pour gérer le stress (Le Scanff, 2003) ou qu'un haut niveau de cohésion dans les groupes sportifs était associé à une diminution de l'anxiété situationnelle.

L'importance des relations avec les partenaires et la cohésion du groupe sont mises en avant par les étudiants circassiens dans leur gestion de la peur et du risque, en particulier dans les spécialités nécessitant de travailler en duo (un porteur et un voltigeur en main à main et en cadre aérien) ou en collectif (bascule coréenne). Les collectifs, tout comme les duos, peuvent être considérés comme des groupes sportifs en se référant à la définition 


\section{eJRIEPS 35 avril 2015}

de Carron et Hausenblas (1998) : "rassemblement de deux ou plusieurs individus qui possèdent une identité commune, ont des buts et des objectifs communs, partagent un destin commun, présentent des patrons structurés dinteraction et de communications, possèdent des perceptions communes de la structure du groupe, sont personnellement et instrumentalement interdépendants, manifestent une attirance interpersonnelle réciproque et se considèrent eux-mêmes comme un groupe " (p.13-14). Cependant, les caractéristiques de ces duos et collectifs se différencient des groupes habituellement étudiés qui sont essentiellement des équipes de sport collectif (où les tâches interactives nécessitent une grande coordination des actions pour produire une performance collective) et dans une moindre mesure des équipes de sport individuel (où les tâches sont co-actives avec une performance collective obtenue par addition des performances individuelles). Si les tâches des circassiens sont effectivement interactives et nécessitent une grande coordination afin de produire la performance, la mise en jeu de l'intégrité physique du voltigeur qui se trouve entre les mains de son porteur, ajoute une dimension de premier ordre à cette relation qu'on ne retrouve pas dans les sports collectifs. Or, à notre connaissance, aucune recherche n'a pris en compte cette particularité, ce qui pourrait expliquer que la confiance en l'autre est une variable qui n'est pas mise en avant dans les études sur la cohésion d'un groupe sportif. Pourtant dans les discours des étudiants circassiens, la cohésion importante entre les partenaires semble fortement liée à la confiance en l'autre, tant à un niveau opératoire que social. Les voltigeurs l'illustrent très bien (« J'étais tellement en confiance avec lui et j'ai grandi avec lui... Je savais qu'il me rattraperait, que je pouvais faire n'importe quoi..., que ce soit dans notre discipline ou que ce soit à l'extérieur "), mais l'importance de la cohésion et de la confiance dans les collectifs de bascule nous semble encore plus emblématique. La bascule est la spécialité la plus dangereuse et la plus contraignante, il semble que la force du collectif soit puisée dans une très forte cohésion et confiance interindividuelle, une fois de plus tout autant à un niveau opératoire que social. L'exemple d'un des collectifs composés de cinq voltigeurs qui se connaissent depuis de nombreuses années (plus de dix ans pour certains) est révélateur : "Ca dépend vraiment des autres c'est pour ça qu'il faut vraiment qu'il y ait une bonne entente dans le groupe. Nous on a commencé, les cinq, parce qu'on s'entendait vraiment bien, on s'est dit, on va faire un truc. La bascule, c'est marrant, on essaye ça. Et vu qu'on était bien soudé déjà à la base, ça a tout de suite marché à fond, on a l'habitude de se voir tout le temps ". 


\section{eJRIEPS 35 avril 2015}

Cette cohésion opératoire, basée sur une très forte confiance en l'autre à un niveau technique, peut être liée à une cohésion sociale importante, une entente parfois très spontanée («On a de la chance... depuis le début on s'entend super bien. Et euh, on s'écoute bien, je pense... Tu peux juste pas pratiquer, si t'es pas ensemble et si t'es pas à l'écoute de l'autre "). Parfois la cohésion opératoire se construit principalement à partir du travail technique («Me dire que lui il fait son job, que moi je fais mon job, et que du coup cette confiance elle se créera petit à petit ... Que lui il aura pris confiance en lui, que moi j'aurai pris confiance en moi et que du coup on pourra avoir confiance en nous deux "), mais une fois installée cette cohésion opératoire pourra, à l'inverse, influencer la cohésion sociale («Y'a une relation qui se crée... et après y a une relation amicale, relation professionnelle et c'est une vraie relation de confiance »). Un seul étudiant a évoqué un apprentissage plus formel de la confiance en l'autre sous forme de cours ponctuels de « conscience collective » dans une école préparatoire (e.g., se laisser tomber en arrière et rattraper par les autres).

L'émulation du groupe à l'entrainement favorise également une certaine gestion de la peur et de la prise de risque. La comparaison aux autres, le regard des autres, voir les autres y arriver ou vouloir montrer aux autres que l'on peut y arriver permet à l'étudiant d'aller audelà de ses appréhensions (« ce qui me pousse à faire les choses, c'est aussi l'émulation collective »). Cependant si l'émulation collective permet aux élèves de gérer leur peur et de se lancer plus facilement, elle peut aussi être source de danger notamment pendant les pauses et, ou quand les enseignants ne sont pas présents (Legendre, 2014).

Finalement, les dimensions interindividuelles de gestion de la peur et du risque des étudiants sont surtout basées sur la confiance en l'autre et ce, au niveau de deux pôles en interaction: l'un technique et l'autre affectif. Avec l'enseignant, la confiance est très importante que ce soit dans la progression technique proposée aux étudiants ou pour se lancer dans des figures difficiles. L'expertise de l'enseignant (pôle technique) est donc à la base de la confiance des étudiants (pôle affectif). Avec les partenaires, la cohésion et la confiance se retrouvent également à un niveau technique (chacun doit faire «son boulot »), qui suit ou précède une cohésion sociale (plus affective) également importante.

\section{Conclusion}

Cette étude exploratoire a permis de mettre en avant l'importance des dimensions personnelles intra-individuelles et interindividuelles, perçues par les étudiants en école supérieure de cirque, dans leur gestion du risque corporel et son apprentissage. 


\section{eJRIEPS 35 avril 2015}

Les dimensions intra-individuelles concernent la gestion de la peur, la concentration et la confiance en soi et en son corps. Cette notion de confiance est également à la base de la gestion de la peur et du risque à un niveau interindividuel, tant à un niveau technique qu'affectif. A un niveau opératoire, les étudiants font confiance à leurs enseignants, experts reconnus de leur discipline, pour tout ce qui concerne les apprentissages techniques. Cette expertise se décline à un niveau plus affectif, quand l'enseignant encourage l'étudiant à se lancer dans une nouvelle figure car il considère que c'est le « bon moment », l'étudiant lui fait alors totalement confiance. Les étudiants font également confiance, spontanément ou de façon construite, techniquement et d'une façon plus générale, à leur(s) partenaire(s). Cette cohésion tant opératoire que sociale, voire affective, permet le développement d'une véritable symbiose notamment entre le voltigeur et son porteur.

Au niveau des apprentissages de la gestion du risque, il semble qu'un des enjeux majeurs de la formation sera de faire évoluer la peur du profane vers le risque maitrisé du professionnel. L'émotionnel sera alors rationnalisé avec la maitrise progressive des techniques tant mentales que corporelles. En effet, la gestion de la peur et la concentration passent par un apprentissage informel de techniques personnelles qui s'apparentent aux routines de performance (favoriser un déroulement automatique du mouvement en essayant de ne pas trop réfléchir, discours interne pour se rassurer). Cet apprentissage semble pertinent, mais gagnerait en efficacité et en rapidité s'il était rationalisé et formalisé (préparation mentale notamment). La connaissance et la confiance en soi et dans son corps vont être développées indirectement et essentiellement par la maitrise (voire l'automatisation) issue d'un apprentissage formel et répétitif des techniques corporelles et des réchappes. La confiance dans l'enseignant et dans les partenaires se développe aussi sur la base de dimensions techniques: l'expertise de l'enseignant rassure les étudiants, tout comme la maitrise technique des partenaires. Cependant, les dimensions techniques sont aussi en interaction avec les dimensions affectives, elles s'influencent mutuellement: la cohésion sociale peut précéder ou suivre la cohésion opératoire, la confiance dans l'enseignant vient notamment de son expertise technique, l'enseignant qui connait son étudiant sait adapter ses enseignements techniques, etc. Ceci conforte le point de vue de Saury (2004) qui remet en question les deux pôles distincts « relationnel ou affectif » versus « instructif » dans la relation ou collaboration entraineursportif, les deux semblent bien interdépendants. 


\section{eJRIEPS 35 avril 2015}

La gestion du risque dans le milieu du cirque est souvent réduite à la maitrise technique. Finalement, s'il semble bien que l'apprentissage des techniques sportives soit la pierre angulaire de la gestion du risque, notamment parce qu'il participe largement au développement de dimensions personnelles indispensables à cette gestion : la confiance en soi, en son corps et en l'autre. L'autre apprentissage qui semble primordial dans la gestion du risque, et qui mériterait d'être formalisé, concerne les techniques mentales de gestion de la peur. Par ailleurs, dans les écoles supérieure de cirque, les étudiants perçoivent l'apprentissage de la gestion du risque comme quelque chose qui est bien présent mais de façon diffuse et informelle: «On n'a pas un cours pour la gestion du risque... ça va être travaillé petit à petit... on ne se rend pas forcément compte qu'on le travaille, mais on le travaille... on n'a pas une ampoule rouge qui fait "gestion du risque, c'est un risque, faut faire attention ! ". C'est toujours, c'est tout le temps, c'est tout le temps là et on le sait... on est tout le temps en train de gérer ce truc-là ».

Cette étude exploratoire a permis de mettre en avant les thèmes qui étaient les plus abordés par les étudiants en école supérieure de cirque concernant leur gestion personnelle du risque et son apprentissage. Ces thèmes sont évidemment amenés à être approfondis notamment avec des méthodologies plus spécifiques. Les relations interpersonnelles chez les circassiens nous semble, par exemple, un domaine tout à fait intéressant à étudier. En effet, certaines spécialités de cirque mêlent une présence importante de risque corporel et une coopération avec des partenaires qui gèrent une partie de ce risque (main à main, cadre aérien, bascule coréenne). Cette spécificité peu commune dans le sport, permettrait notamment d'approfondir la construction de la confiance et de la cohésion qui oscille entre technique et affectif (entre le voltigeur et son porteur par exemple). Par ailleurs, cette étude a mis aussi en avant la très forte confiance des étudiants envers leur enseignant. II serait alors intéressant d'approfondir comment ces derniers gèrent le risque de leurs étudiants ? Sur quoi se basent-ils ? Qu'est-ce qui justifie cette confiance aveugle des étudiants en leurs enseignants ? Dans cette perspective, il serait notamment pertinent d'étudier en situation la gestion du risque par l'enseignant et son impact sur l'engagement des étudiants.

\section{Bibliographie}

Bandura, A. (1977). Self-efficacy: toward a unifying theory of behavioral change. Psychological review, 84, 191-215. 


\section{eJRIEPS 35 avril 2015}

Bernier, M., Thienot, E., \& Codron, R. (2009). Attention et performance sportive : état de la question en psychologie du sport appliquée. Staps, 1(83), 25-42.

Bonnet, A., Pedinielli, J.L., Romain, F., \& Rouan, G. (2003). Bien-être subjectif et régulation émotionnelle dans les conduites à risque. Cas de la plongée sousmarine. L'Encéphale, 29, 488-497.

Buton, F., Fontayne, P., \& Heuzé, J.P. (2006). La cohésion des groupes sportifs: évolutions conceptuelles, mesures et relations avec la performance. Science \& Motricité, 3(59), 9-45.

Camiolo, M. (2013). Wilde, G., Le risque cible. Une théorie de la santé et de la sécurité. Questions Vives, 9(19), 159-161.

Carron, A. V., \& Hausenblas, H. A. (1998). Group dynamics in sport (2nd. Ed.). Morgantown, WV : Fitness Information Technology.

Carron, A. V., Widmeyer, W. N., \& Brawley, L. R. (1985). The development of an instrument to assess cohesion in sport teams : The Group Environment Questionnaire.Journal of Sport Psychology, 7, 244-266.

Collard, L. (1998). Sports, enjeux et accidents. Paris : PUF.

Collard, L. (2002). Le risque calculé dans le défi sportif. L'Année sociologique, 52, 351369.

Corneloup, J. \& Soulé, B. (2002). La gestion du risque dans les activités sportives de nature. Revue Espaces, 73, 32-52.

Cox, R.H. (2005). Psychologie du sport. Bruxelles : Edition De Boeck.

Crews, D.J., \& Boutcher, N.H. (1986). Effects of structured preshot behaviors on beginning golf performance. Perceptuel and motor skills, 62, 291-294.

Crews, D.J., \& Landers, D.M. (1991). Cardiac pattern as an indication of attention: a test of two hypotheses. Unpublished manuscript, Department of Exercise Science en Physical Education, Arizona State University, Tempe.

Debois, N. (2003). De l'anxiété aux émotions compétitives : état de la recherche sur les états affectifs en psychologie du sport. Staps, 3(62), 21-42.

De Ketele, J.M., \& Rogiers, X. (2009). Méthodologie du recueil d'informations (4 ${ }^{e ̀ m e}$ édition). Bruxelles-Paris : De Boeck Université

Delignières, D. (1991). Risque perçu et apprentissage moteur. In J.P. Famose, P. Fleurance \& Y. Touchard (Eds.), Apprentissage moteur : rôle des représentations (pp. 157-171). Paris: EPS. 


\section{eJRIEPS 35 avril 2015}

Delignières, D. (1993). Risque préférentiel, risque perçu et prise de risque. In J.P. Famose (Ed.), Cognition et performance (pp. 79-102). Paris : INSEP.

Demontrond, P., \& Gaudreau, P. (2008). Le concept de « flow » ou « état psychologique optimal » : état de la question appliquée au sport. Staps, 1(79), 9-21.

Deslauriers, J.P. (1991). Recherche qualitative, guide pratique. Montréal : McGraw-Hill.

Famose, J.P., Guerin, F., \& Sarrazin, P. (2005). Les croyances sur soi : clarification conceptuelle, formation, et relations à la performance sportive. In O. Rascle \& $\mathrm{P}$. Sarrazin (Eds.), Croyances et performance sportive. Processus sociocognitifs associés aux comportements sportifs (pp. 19-52). Paris : Editions Revue EPS

Ferrel-Chapus, C., \& Tahej, P.K. (2010). Processus attentionnels et apprentissage moteur. Movement \& Sport Sciences, 3(71), 71-83.

Fourmaux, F. (2006). Le nouveau cirque ou l'esthétisation du frisson. Ethnologie Française, 36(4), 659-668.

Goudart, P. (2001). Esthétique du risque : du corps sacrifié au corps abandonné. Actes de l'université d'été «L'école en piste, les arts du cirque à la rencontre de l'école », Avignon, France, juillet.

Gould, D. \& Udry, E. (1994). Psychological skills for enhancing performances: arousal, regulation strategies. Medicine and science in sports and exercise, 26(4), 478-485.

Gould, D., Weiss, M.R., \& Weinberg, R. (1981). Psychological characteristics of successful and non-successful big-ten wrestels. Journal of sport psychology, 3, 69-81.

Guibert, J. \& Jumel, G. (1997). Méthodologie des pratiques de terrain en sciences humaines et sociales. Paris : Armand Colin.

Heuzé, J.P. (2003). Le leadership. In C. Le Scanff (Ed.), Manuel de psychologie du sport : l'intervention auprès du sportif (pp. 315-342). Paris : Editions Revue EPS.

Jowett, S., \& Meek, G. A. (2000). The coach-athlete relationship in married couples: An exploratory content analysis. The Sport Psychologist, 14, 157-175.

Kouabenan, D. R. (2006). Des facteurs structurants aux biais ou illusions dans la perception des risques. In D. R. Kouabenan, B. Cadet, D. Hermand \& M.T. Munoz Sastre (Eds.), Psychologie du risque : identifier, évaluer, prévenir (pp. 125-145). Bruxelles : De Boeck \& Larcier.

Lafollie, D., \& Le Scanff, C. (2007). Détection des personnalités à risque dans les sports à sensations fortes. L'Encéphale, 33(2), 135-141. 


\section{eJRIEPS 35 avril 2015}

Lafollie, D., \& Le Scanff, C. (2008). Recherche de sensations, désinhibition et pratique de sports à risque: quelques pistes de réflexion. Annales Médico-psychologiques, revue psychiatrique, 166(10), 794-798.

Legendre, F. (2014). La transmission de la gestion du risque dans les écoles supérieures de cirque en France. SociologieS [En ligne], Dossiers, La transmission du métier. (en ligne), 02 juin 2014. http://sociologies.revues.org/4554

Le Scanff, C. (1999). Une approche multidimensionnelle de la gestion du stress. In C. Le Scanff \& J.P. Famose (Eds.), La gestion du stress. Entrainement et compétition (pp. 18-25). Paris : Editions Revue EPS.

Le Scanff, C. (2003). La gestion du stress. In C. Le Scanff (Ed.), Manuel de psychologie du sport: l'intervention auprès du sportif (pp. 121-148). Paris: Editions Revue EPS.

Le Scanff, C., \& Famose, J.P. (1999). La gestion du stress. Entrainement et compétition. Dossier $n^{\circ} 43$. Paris : Editions Revue EPS.

Le Scanff, C., \& Nicchi, S. (2004). La personnalité. In J. LaRue, \& H. Ripoll (Eds.), Manuel de psychologie du sport: les déterminants de la performance sportive (pp. 411428). Paris : Editions Revue EPS.

Le Scanff, C., \& Nicchi, S. (2004). Les stratégies de coping. In J. LaRue, \& H. Ripoll (Eds.), Manuel de psychologie du sport: les déterminants de la performance sportive (pp. 475-512). Paris : Editions Revue EPS.

Lévêque, M. (1995). Coach athlete relationship: a clinical perspective. Actes du 9th European Congress on Sport Psychology. Bruxelles.

Losier, G.F., \& Vallerand, R.J. (1995). Développement et validation de l'Echelle des Relations Interpersonnelles dans le Sport (ERIS). International Journal of Sport Psychology, 26, 307-326.

Martin-Krumm, C., \& Sarrazin, P. (2004). Théorie des styles explicatifs et performance sportive : fondements théoriques, données empiriques et perspectives. Movement \& Sport Sciences, 2(52), 9-43.

Masters, R.S.W. (1992). Knowledge, knerves and knowhow: the role of explicit versus implicit knowledge in the breakdown of a complex motor skill under pression. British Journal of Psychology, 83, 343-358.

Meichenbaum, D.H. (1977). Cognitive behavior modification: an integrated approach. New-York : Plenum. 


\section{eJRIEPS 35 avril 2015}

Michel, G. (2001). La prise de risque à l'adolescence : Pratique sportive et usage de substances psycho-actives. Paris : Masson.

Michel, G., Purper-Ouakil, D., \& Mouren-Siméoni, M.C. (2002). Prises de risques chez les jeunes. Les conduites dangereuses en véhicules motorisées. Neuropsychiatrie de l'enfance et de l'adolescence, 50, 583-589.

Paillé, P., \& Mucchielli, A. (2012). L'analyse qualitative en sciences humaines et sociales. Paris : Armand Colin.

Perreaut-Pierre, E. (2000). Gestion mentale du stress pour la performance sportive. Paris : Amphora.

Pion, J., \& Raimbault, N. (2008). La préparation mentale en sports individuels. Paris: Chiron Editeur.

Raveneau, G. (2006). La plongée sous-marine, entre neutralisation du risque et affirmation de la sécurité. Ethnologie Française, 36(4), 613-623.

Ripoll, H. (2004). Les opérations cognitives en contexte sportif. In J. LaRue, \& H. Ripoll (Eds.), Manuel de psychologie du sport: les déterminants de la performance sportive (pp. 151-296). Paris : Editions Revue EPS.

Ripoll, H. (2008). Le mental des champions. Comprendre la réussite sportive. Paris: Payot.

Saury, J. (2004). L'entrainement comme système d'aide à la performance. In J. Saury, \& C. Sève (Eds.). L'entrainement : les entraineurs et leurs pratiques, (pp. 51-65).

Saury, J., \& Durand, M. (1995). De l'analyse des relations entraineurs-athlètes à une modélisation de la situation d'entrainement comme un travail collectif. Sport, 151, 25-39.

Shiffrin, R.M., \& Schneider, W. (1977). Controlled and automatic human information processing : II. Perceptual learning, automatic attending, and a general theory. Psychological Review, 84, 127-190.

Singer, R.N. (2002). Preperformance state, routines, and automaticity: what does it take to realize expertise in self-paced events? Journal of Sport and Exercise Psychology, 24, 359-375.

Sizorn, M. (2008). Une ethnologue en Trapézie : sport, art ou spectacle ? Ethnologie Française, 1, 79-88.

Soulé, B. \& Corneloup, J. (1998). Jeunes et prises de risque sportives. Vers une approche sociologique contextualisée. Corps \& Culture, 3, 107-130. 


\section{eJRIEPS 35 avril 2015}

Soulé, B., \& Corneloup, J. (2007). Sociologie de l'engagement corporel. Risques sportifs et pratiques «extrêmes » dans la société contemporaine. Paris : Armand Colin.

Totchilova-Gallois, E. (2005). La relation entraineur-entrainé en sport de haut niveau: analyse du concept de mentoring. Thèse de doctorat non publiée, Université d'Orléans, Orléans.

Wilde, G.J. (1988). Risk homeostasis theory and traffic accidents: propositions, deductions and discussion of dissension in recent reactions. Ergonomics, 31, 441-468.

Wilde, G.J. (2012). Le risque cible. Une théorie de la santé et de la sécurité. Fernelmont : InterCommunication.

Wulf, G., \& Prinz, W. (2001). Directing attention to movement effects enhances learning: A review. Psychonomic Bulletin \& Review, 9(2), 185-211.

Zuckerman, M. (2006). Sensation seeking and risky behavior. Washington, DC: American Psychological Association. 